\title{
Malignancy mimicking Juvenile Nasopharyngeal Angiofibroma
}

\author{
${ }^{1}$ Navjot Kaur, ${ }^{2}$ Harsimran Tiwana, ${ }^{3}$ Shikhar Sawhney, ${ }^{4}$ Ashok K Gupta
}

\begin{abstract}
Nasopharyngeal masses in young males can be benign or malignant. As the treatment regimen for these masses differs considerably, it is crucial to differentiate benign from malignant masses for successful and adequate treatment of patients and also for minimizing morbidity from unnecessary interventions. We are presenting two case reports of patients with nasopharyngeal carcinoma (NPC) mimicking juvenile nasopharyngeal angiofibroma (JNA). Both the cases were presumed as JNA and underwent excessive surgical intervention only to reveal NPC on final histopathology report. We wish to highlight the importance of complete clinical examination and preoperative imaging in differentiating an ideal management of nasopharyngeal masses.
\end{abstract}

Keywords: Carcinoma, Endoscopic approach to juvenile nasopharyngeal angiofibroma, Nasopharynx.

How to cite this article: Kaur N, Tiwana H, Sawhney S, Gupta AK. Malignancy mimicking Juvenile Nasopharyngeal Angiofibroma. Clin Rhinol An Int J 2017;10(2):99-102.

Source of support: Nil

Conflict of interest: None

\section{INTRODUCTION}

Juvenile nasopharyngeal angiofibromas (JNA) are rare benign tumors presenting in male adolescents as nasal obstruction and epistaxis. Although these tumors are benign, they are highly vascular and locally aggressive. Biopsy or surgical resection prior to imaging, angiography, or embolization may be hazardous. The definitive treatment is surgical resection.

Children and adolescent nasopharyngeal carcinoma (NPC) is very rare worldwide. ${ }^{1}$ These patients usually present with advanced locoregional disease and high prevalence of distant metastasis. ${ }^{2,3}$ The mainstay of treatment is external beam radiotherapy, which is considered as the treatment of choice. ${ }^{4}$

\footnotetext{
1,2 Senior Resident, ${ }^{3}$ Junior Resident, ${ }^{4}$ Professor and Head

${ }^{1-4}$ Department of ENT, Postgraduate Institute of Medical Education \& Research, Chandigarh, India

Corresponding Author: Ashok K Gupta, Professor and Head, Department of ENT, Postgraduate Institute of Medical Education \& Research, Chandigarh, India, Phone: +919814198850 e-mail: drashokpgi@hotmail.com
}

We present two cases of adolescent males with a nasopharyngeal mass that presented with physical and radiographic findings suggesting JNA. Considering this diagnosis, biopsy was not done. Both patients underwent excessive surgical intervention which could have been avoided. Histopathology revealed NPC.

\section{CASE REPORTS}

\section{Case 1}

An 18-year-old boy presented to the ear, nose and throat (ENT; Otolaryngology-Head Neck Surgery) outpatient services of Postgraduate Institute of Medical Education \& Research, Chandigarh India, with 1 year history of recurrent episodes of epistaxis, right side nasal obstruction, and pain over right side of face. It was also associated with reduced sensation over right side of face and reduced mouth opening for the last 8 months. A contrastenhanced computed tomography (CT) scan was obtained which showed a homogeneously enhancing soft-tissue mass lesion in the nasopharynx centered around the sphenopalatine foramen. The mass was seen extending into the pterygopalatine fossa, causing anterior bowing of the posterior wall of the right maxillary sinus. Superiorly, it was extending into the middle cranial fossa through the widened foramen ovale. It had also invaded into bilateral sphenoid sinuses and laterally it was extending into the infratemporal fossa. Magnetic resonance imaging was also done to see its intracranial and intraorbital extension (Fig. 1).

The patient underwent endoscopic endonasal surgical resection. Intraoperatively, firm fleshy mass was seen involving right nasal cavity; posteriorly, it was extending into the nasopharynx. Superiorly, it was eroding the roof of sphenoid sinus and laterally extending into the infratemporal fossa with erosion of skull base and intracranial extension. The mass was removed. Histopathological examination revealed moderately differentiated squamous cell carcinoma. The patient was subsequently referred for chemoradiation.

\section{Case 2}

A 23-year-old adolescent male presented to the ENT (Otolaryngology-Head Neck Surgery) outpatient services of Postgraduate Institute of Medical Education \& Research, 

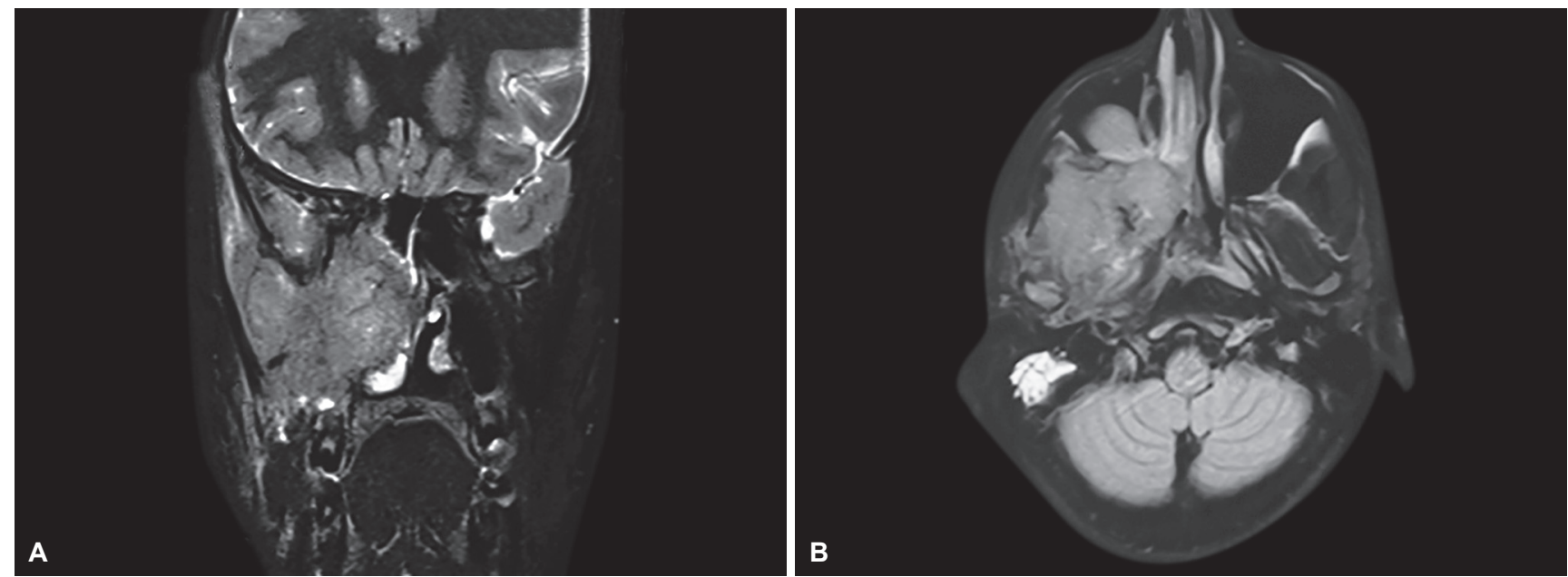

Figs $1 \mathrm{~A}$ and $\mathrm{B}$ : Magnetic resonance imaging: (A) coronal; and (B) axial view respectively, showing the tumor extending to pterygopalatine fossa causing anterior bowing of posterior wall maxillary sinus, mimicking angiofibroma

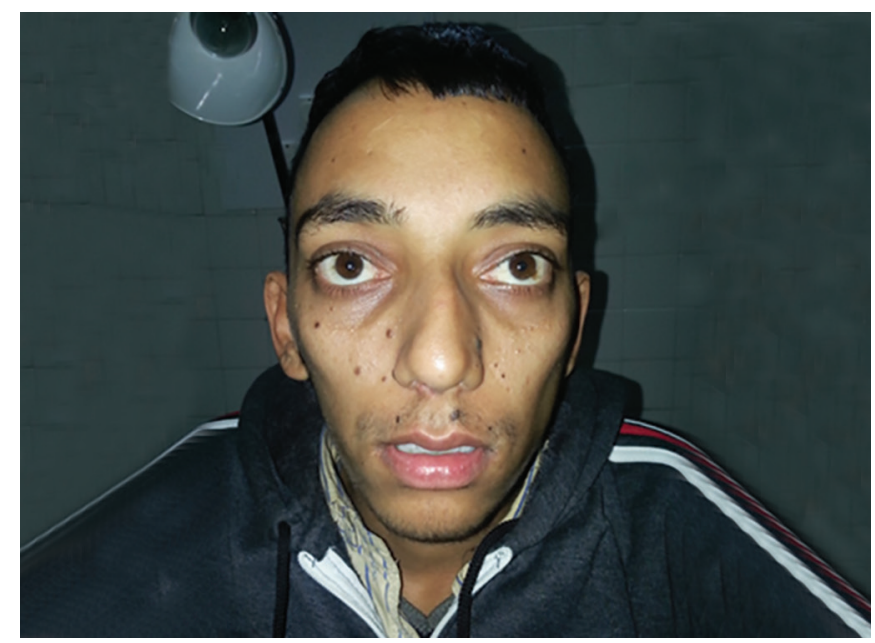

Fig. 2: Clinical view of deviated nasal septum toward left side with left eye proptosis (case 2)

Chandigarh, India, with 3 months history of left side unilateral nasal obstruction and recurrent episodes of epistaxis. The ENT examination showed deviated nasal septum toward left side with left eye proptosis (Fig. 2); endoscopic examination showed a reddish, smoothsurfaced, firm fleshy mass in the left nasal cavity. Contrast-enhanced CT scan showed lobular heterogeneously enhancing soft-tissue density mass in left nasal cavity, nasopharynx, and pterygopalatine fossa with extension along the posteromedial aspect of left maxillary sinus. There was no bone destruction. It also revealed presence of a lymph node on right level II.

Provisional diagnosis of JNA was made. The patient was operated upon via endoscopic endonasal approach and the mass was removed (Figs 3 and 4).

There was around $300 \mathrm{~mL}$ of blood loss and this tumor was more vascular than was expected. Histology showed NPC. Patient was subsequently referred for chemoradiation.

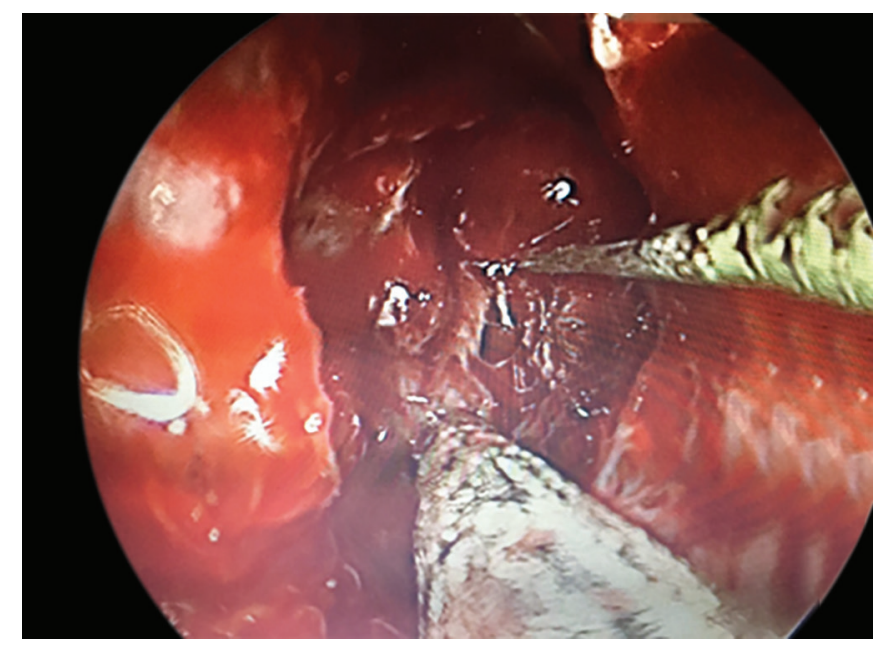

Fig. 3: Intraoperative view with the mass filling the sphenoid sinus (case 2)

\section{Case 3}

A 28-year-old young male was referred to the ENT department outpatient services from a periphery hospital, Uttarakhand, India, with a diagnosis of angiofibroma. He had a 5-month history of recurrent nasal bleed, diplopia from left eye with left eye squint, and left side nasal obstruction for the last 2 months. Nasal endoscopy examination showed firm, fleshy mass, bleeding on touch occupying left nasal cavity (Fig. 5) and lateral rectus palsy. Contrast-enhanced CT scan showed heterogeneously enhancing soft-tissue density mass in left nasal cavity, nasopharynx, pterygopalatine fossa, sphenoid sinus, and cavernous sinus with minimal skull base erosion. Although clinical and radiological examination were also creating suspicion for JNA, due to features like skull base erosion, lateral rectus palsy, a provisional diagnosis of NPC was made. Nasal biopsy was taken and was sent for histopathological examination which came out as NPC. Patient was referred for chemoradiation. 


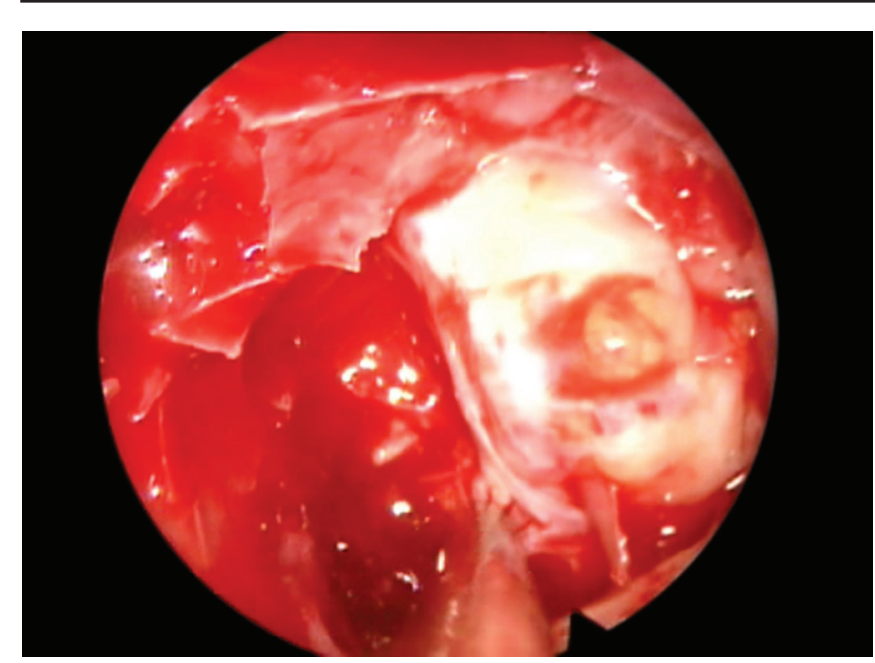

Fig. 4: Intraoperative view after tumor removal (case 2)

\section{DISCUSSION}

Juvenile nasopharyngeal angiofibroma is a benign vascular tumor of nasopharynx and accounts for less than $1 \%$ of all head and neck tumors and usually occurs in adolescent males. It is a locally infiltrative, slow-growing vascular tumor arising in the area of sphenopalatine foramen at the root of pterygoid process on the lateral nasal wall or from the vidian canal area. ${ }^{5}$ They initially expand adjacent structures and will eventually erode bone and invade surrounding structures. They expand laterally into the infratemporal fossa via pterygopalatine fossa; anteriorly into the nasopharynx, chaonal space, anterior nasal cavity, and all the related sinuses and can have intracranial extensions. Juvenile nasopharyngeal angiofibroma patients commonly present with nasal obstruction, epistaxis, blood-stained sputum, and serous otitis media with rubbery vascular nasopharyngeal mass on examination. A CT scan with contrast will show an enhancing soft-tissue mass arising from the nasopharynx or lateral nasal wall. ${ }^{6}$ The pathognomonic radiological feature of JNAs is the anterior bowing of the posterior maxillary wall (Holman-Miller sign). Other features may include a mass originating at the sphenopalatine foramen with widening and erosion of the pterygomaxillary fossa, sphenoid sinus, and infratemporal fossa. ${ }^{7}$ Site of origin, hypervascularization after contrast enhancement, and patterns of growth are three factors which form the basis of diagnosis on imaging. ${ }^{5,8}$ Biopsy is absolutely contraindicated because of risk of massive hemorrhage. ${ }^{9}$ Further confirmation of the JNA diagnosis is usually provided by angiography, which also doubles as treatment with embolization and gives information on specific blood supply of the tumor. ${ }^{7}$

Thus, the diagnosis of JNA is based on clinical and radiological examination and the final proof of diagnosis is histologic. ${ }^{6}$

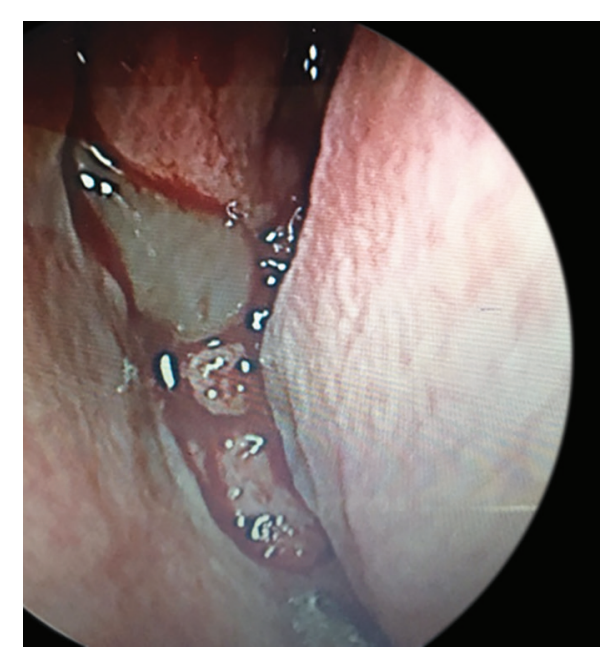

Fig. 5: Nasal endoscopic view with pinkish pale polypoidal mass filling the left nasal cavity with blood clots (case 3)

Nasopharyngeal carcinoma, although rare, in children has a predilection for adolescents. Children aged between 10 and 19 years are the most common age group affected. Childhood NPC is believed to have a better diagnosis than the adult type. The diagnosis is usually delayed and the patients present at a locally advanced stage. In addition to the nonspecificity of the symptoms, not considering the entity for differential diagnosis might be the other reason for a delayed diagnosis. Computed tomography being accessible, fast, accurate, relatively cheap, and noninvasive is frequently used for the diagnosis of NPC. On imaging, NPC appears as a heterogeneously enhancing mass arising from the nasopharyngeal mucosa or submucosal space with skull base erosion and invasion into the surrounding structures due to its malignant nature. Computed tomography features may show nasopharyngeal or oropharyngeal asymmetry, obliteration of pharyngeal opening of Eustachian tube, obliteration of Rosenmüller fossa, asymmetry of piriform recesses, extension into nasal cavity, skull base involvement, mastoid opacification, sphenoid sinus or orbital invasion, extension into pterygopalatine fossa and widening it, parapharyngeal, masticator spaces or petroclival fissures involvement, presence of unilateral or bilateral cervical lymphadenopathy, or presence of lateral retropharyngeal lymphadenopathy. Cervical lymphadenopathy is frequently evident. ${ }^{10}$

These three cases are presented to highlight the salient imaging features that should not be overlooked for evaluation and diagnosis of JNA and NPC and the importance of detailed clinical examination. Case 1 shows a homogeneously enhancing soft-tissue nasopharyngeal mass centered around sphenopalatine foramen with extension into the pterygopalatine fossa and causing anterior bowing of the posterior wall of the right maxillary sinus, also extending into middle cranial 
fossa, bilateral sphenoid sinus, and infratemporal fossa. All these features indicate toward JNA, but histopathology revealed NPC as the final diagnosis. In this case, although there was evidence of a Holman-Miller sign, this finding is not a unique feature of JNA and other features should not be overlooked. Case 2 showed a lobular heterogeneously enhancing soft-tissue density mass in left nasal cavity, nasopharynx, and pterygopalatine fossa with extension along posteromedial aspect of left maxillary sinus. Specifically, imaging also revealed the presence of lymphadenopathy in right level II. Case 3 also showed heterogeneously enhancing soft-tissue density mass involving left nasal cavity, nasopharynx, pterygopalatine fossa, sphenoid sinus, cavernous sinus with minimal skull base erosion, and a lymph node left level II.

Because $90 \%$ of JNAs present with involvement of the pterygopalatine fossa and very typically homogeneously enhance, isolated heterogeneous masses arising from the nasopharyngeal mucosa without involvement of the sphenopalatine foramen and features like muscle invasion and the presence of cervical lymphadenopathy should prompt consideration for entities, such as NPC.

\section{CONCLUSION}

It is imperative that preoperative imaging of patients with presumed JNA be carefully reviewed to ensure accurate diagnosis and proper management. Uncommon features seen on imaging, such as the lack of involvement of the pterygopalatine fossa, the presence of soft-tissue invasion, and cervical lymphadenopathy should alert the physician to malignant etiologies, such as NPC, and the radiologist must be aware that NPC can occur in children and, therefore, should deliberately look for associated secondary diagnostic signs, and the clinician should be able to correlate clinical and radiological findings for making an accurate diagnosis.

\section{REFERENCES}

1. Liu W, Tang Y, Gao L, Huang X, Luo J, Zhang S, Wang K, Qu Y, Xiao J, Xu G, et al. Nasopharyngeal carcinoma in children and adolescents - a single institution experience of 158 patients. Radiat Oncol 2014 Dec 5;9:274.

2. SultanI,Casanova M,FerrariA, RihaniR,Rodriguez-GalindoC. Differential features of nasopharyngeal carcinoma in children and adults: a SEER study. Pediatr Blood Cancer 2010 Aug;55(2):279-284.

3. Downing NL, Wolden S, Wong P, Petrik DW, Hara W, Le QT. Comparison of treatment results between adult and juvenile nasopharyngeal carcinoma. Int J Radiat Oncol Biol Phys 2009 Nov 15;75(4):1064-1070.

4. Daoud J, Toumi N, Bouaziz M, Ghorbel A, Jlidi R, Drira MM, Frikha M. Nasopharyngeal carcinoma in childhood and adolescence. Eur J Cancer 2003 Nov;39(16):2349-2354.

5. Lloyd G, Howard D, Lund VJ, Savy L. Imaging for juvenile angiofibroma. J Laryngol Otol 2000 Sep;114(9):727-730.

6. Nicolai P, Castelnuovo P. Benign tumors of the sinonasal tract. In: Flint PW, Haughey BH, Lund VJ, Niparko JK, Robbins KT, Thomas JR, Lesperance MM, editors. Cummings otolaryngology head and neck surgery. 6th ed. Philadelphia (PA): Elsevier Saunders; 2015. p. 744-746.

7. Blount A, Riley KA, Woodworth BA. Juvenile nasopharyngeal angiofibroma. Otolaryngol Clin 2011 Aug;44(4):989-1004.

8. Maroldi R, Nicolai P. Imaging in treatment planning for sinonasal diseases. Berlin: Springer; 2004.

9. Lund VJ, Stammberger $\mathrm{H}$, Nicolai $\mathrm{P}$, Castelnuovo $\mathrm{P}$, Beal $\mathrm{T}$, Beham A, Bernal-Sprekelsen M, Braun H, Cappabianca P, Carrau R, et al. European position paper on endoscopic management of tumours of the nose, paranasal sinuses and skull base. Rhinol Suppl 2010 Jun 1;(22):1-143.

10. Eksioglu AS, Çinar HG, Ucan B, Bozkurt C, Ankara TR. Nasopharyngeal carcinoma: Computed Tomography features in children and adolescents. European Society of Radiology. p. $1-28$. 